Proyecciones

Vol. 28, $\mathrm{N}^{o}$ 1, pp. 47-56, May 2009.

Universidad Católica del Norte

Antofagasta - Chile

DOI: $10.4067 /$ S0716-09172009000100005

\title{
FINITISTIC SPACES IN L-TOPOLOGICAL SPACES
}

\author{
T. BAIJU \\ and \\ SUNIL JACOB JOHN \\ NATIONAL INSTITUTE OF TECHNOLOGY CALICUT, INDIA \\ Received: February 2009. Accepted: April 2009
}

\begin{abstract}
In this paper the concept of finitistic spaces in $L$-topological spaces is introduced by means of $\alpha$-Q-covers of open $L$ subsets. Also a characterization of finitistic spaces in the weakly induced L-topological spaces is obtained. Moreover the behavior of finitistic spaces under various types of maps such as fuzzy perfect maps is also investigated.
\end{abstract}




\section{Introduction}

Fuzzy mathematics begins with Zadeh's paper of 1965, has come of age. The language and behavior of it have developed accordingly. The concept of finitistic spaces in general topology was introduced by R. G. Swan [16] in 1960. But the term finitistic was used by Bredon [2]. Many results related to finitistic spaces also known as boundedly metacompact spaces can be seen in $[4,5,6,7,9,10]$. In [12], Jamwal and Shakeel introduced the concept of finitistic spaces in fuzzy topological spaces by using the usual open cover in $L$-fuzzy topology. In [1], authors have introduced the concept of covering dimension in $L$-topological spaces using Quasi-coincidence relation and $\alpha$-Q-covers and obtained a characterization for it. Motivated by this definition of local covering dimension in $L$-topological spaces, in this paper we introduce the concept of finitistic spaces in $L$-topological spaces using $\alpha$ - $Q$-covers and will study its various properties. Besides getting a characterization for finitistic spaces in the weakly induced $L$-topological spaces, it is also shown that every fuzzy paracompact space with finite covering dimension is finitistic and finitistic property is preserved by fuzzy perfect maps.

\section{Preliminaries and basic definitions}

Let $L$ be a complete lattice. Its universal bounds are denoted by $\perp$ and $\top$. We presume that $L$ is consistent. i.e., $\perp$ is distinct from $T$. Thus $\perp \leq \alpha \leq \top$ for all $\alpha \in L$. We note $\vee \phi=\perp$ and $\wedge \phi=\top$. The two point lattice $\{\perp, \top\}$ is denoted by 2. A unary operation ' on $L$ is a quasicomplementation. It is an involution (i.e., $\alpha^{\prime \prime}=\alpha$ for all $\alpha \in L$ ) that inverts the ordering. (i.e., $\alpha \leq \beta$ implies $\left.\beta^{\prime} \leq \alpha^{\prime}\right)$. In $\left(L,^{\prime}\right)$ the DeMorgan laws hold: $(\vee A)^{\prime}=\wedge\left\{\alpha^{\prime}: \alpha \in A\right\}$ and $(\wedge A)^{\prime}=\vee\left\{\alpha^{\prime}: \alpha \in A\right\}$ for every $A \subset L$. Moreover, in particular, $\perp^{\prime}=\top$ and $\top^{\prime}=\perp$.

A molecule or co-prime element in a lattice $L$ is a join irreducible element in $L$ and the set of all non zero co-prime elements of $L$ is denoted by $M(L)$. Also we denote $A_{(\alpha)}=\{x \in X: A(x) \not \leq \alpha\}$ and $A_{[\alpha]}=\{x \in X: A(x) \geq \alpha\}$.

A complete lattice $L$ is completely distributive if it satisfies either of the logically equivalent CD1 or CD2 below: CD1: $\wedge_{i \in I}\left(\vee_{j \in J_{i}} a_{i, j}\right)=$ $\vee_{\varphi \in \prod_{i \in I} J_{i}}\left(\wedge_{i \in I} a_{i, \varphi_{(i)}}\right)$

$\mathrm{CD} 2: \quad \vee_{i \in I}\left(\wedge_{j \in J_{i}} a_{i, j}\right)=\wedge_{\varphi \in \prod_{i \in I} J_{i}}\left(\vee_{i \in I} a_{i, \varphi_{(i)}}\right)$ for all $\left\{\left\{a_{i j}: j \in J_{i}\right\}: i \in\right.$ $I\} \subset P(L) \backslash\{\phi\}, I \neq \phi$. 
If $\left(L,^{\prime}\right)$ is a complete lattice, then for a set $X, L^{X}$ is the complete lattice of all maps from $X$ into $L$, called $L$-sets or $L$-subsets of $X$. Under point-wise ordering, $a \leq b$ in $L^{X}$ if and only if $a(x) \leq b(x)$ in $L$ for all $x \in X$. If $A \subset X, 1_{A} \in 2^{X} \subset L^{X}$ is the characteristic function of $A$. The constant member of $L^{X}$ with value $\alpha$ is denoted by $\alpha$ itself. We use the same notation to represent crisp set as well as its characteristic function. Wang [17] proved that a complete lattice is completely distributive if and only if for each $\alpha \in L$, there exists $B \subseteq L$ such that (i) $a=\vee A$ and (ii) if $A \subseteq L$ and $a \leq \vee B$, then for each $b \in B$, there exists $c \in A$ such that $b \leq c . \quad B$ is called the minimal set of $a$ and $\beta(a)$ denote the union of all minimal sets of $a$. Again $\beta^{*}(a)=\beta(a) \cap M(L)$. Clearly $\beta(a)$ and $\beta^{*}(a)$ are minimal sets of $a$.

Clearly $L^{X}$ has a quasi complementation ' defined point-wisely $\alpha^{\prime}(x)=\alpha(x)^{\prime}$ for all $\alpha \in L$ and $x \in X$. Thus the DeMorgan laws are inherited by $\left(L^{X},{ }^{\prime}\right)$.

Let $\left(L,^{\prime}\right)$ be a complete lattice equipped with an order reversing involution and $X$ be any non empty set. A subfamily $\tau \subset L^{X}$ which is closed under the formation of sups and finite infs (both formed in $L^{X}$ ) is called an $L$-topology on $X$ and its members are called open $L$-sets. The pair $(X, \tau)$ is called an $L$-topological space $(L-t s)$. The category of all $L$-topological spaces, together with $L$-continuous mappings and the composition and identities of Set is denoted by $L$-Top. Quasi complements of open $L$-sets are called closed $L$-sets.

We know that the set of all non zero co-prime elements in a completely distributive lattice is $\mathrm{V}$-generating. Moreover for a continuous lattice $L$ and a topological space $(X, T), T=i_{L} \omega_{L}(T)$ is not true in general. By proposition 3.5 in Kubiak [13] we know that one sufficient condition for $T=i_{L} \omega_{L}(T)$ is that $L$ is completely distributive.

In [18] Wang extended the Lowen functor $\omega$ for completely distributive lattices as follows: For a topological space $(X, T),(X, \omega(T))$ is called the induced space of $(X, T)$ where $\omega(T)=\left\{A \in L^{X}: \forall \alpha \in M(L), A_{\left(\alpha^{\prime}\right)} \in T\right\}$. In 1992 Kubiak also extended the Lowen functor $\omega_{L}$ for a complete lattice $L$. In fact when $L$ is completely distributive, $\omega_{L}=\omega$.

An $L$-topological space $(X, \tau)$ is called weakly induced space if $\forall \alpha \in$ $M(L), \forall A \in \tau$ it is true that $A^{\left(\alpha^{\prime}\right)} \in[\tau]$ where $[\tau]$ is the set of all crisp open sets in $\tau$.

Based on these facts, in this paper we use a complete, completely distributive lattice $L$ in $L^{X}$. For a standardized basic fixed-basis terminology, we follow Hohle and Rodabaugh [11]. Also $L$-Pnt $(X)$ denote the collection 
of all $L$-fuzzy points in the $L$-ts $(X, \tau)$.

Definition 2.1. [19] Let $(X, \tau),(Y, \mu)$ be $L$-topological spaces, $f: X \rightarrow Y$ be an ordinary mapping. Based on this we define the $L$-fuzzy mapping $f^{\rightarrow}: L^{X} \rightarrow L^{Y}$ and its $L$-fuzzy reverse mapping $f^{\leftarrow}: L^{Y} \rightarrow L^{X}$ by $\mathrm{f} \rightarrow: L^{X} \rightarrow L^{Y}, f^{\rightarrow}(A)(y)=\vee\{A(x): x \in X, f(x)=y\}$ $\forall A \in L^{X}, \forall y \in Y$. $f^{\leftarrow}: L^{Y} \rightarrow L^{X}, f^{\leftarrow}(B)(x)=B(f(x)), \quad \forall B \in L^{Y}, \forall x \in X$.

Definition 2.2. [19] Let $(X, \tau),(Y, \mu)$ be $L$-topological spaces, $f \rightarrow: L^{X} \rightarrow$ $L^{Y}$ an $L$-fuzzy mapping. We say $f^{\rightarrow}$ is an $L$-fuzzy continuous mapping from $(X, \tau)$ to $(Y, \mu)$ if its $L$-fuzzy reverse mapping $f^{\leftarrow}: L^{Y} \rightarrow L^{X}$ maps every open subset in $(Y, \mu)$ as an open one in $(X, \tau)$. i.e., $\forall V \in \mu, f^{\leftarrow}(V) \in \tau$.

Definition 2.3. [19] Let $(X, \tau),(Y, \mu)$ be $L$-topological spaces, $f^{\rightarrow}: L^{X} \rightarrow$ $L^{Y}$ an $L$-fuzzy mapping. We say $f^{\rightarrow}$ is open if it maps every open subset in $(X, \tau)$ as an open one in $(Y, \mu)$. i.e., $\forall U \in \tau, f^{\rightarrow}(U) \in \mu$.

Definition 2.4. [19] Let $(X, \tau),(Y, \mu)$ be $L$-topological spaces, $f \rightarrow: L^{X} \rightarrow$ $L^{Y}$ an $L$-fuzzy mapping. We say $f^{\rightarrow}$ is closed if it maps every closed subset in $(X, \tau)$ as a closed one in $(Y, \mu)$. i.e., $\forall F \in \tau^{\prime}, f^{\rightarrow}(F) \in \mu^{\prime}$.

Definition 2.5. [19] Let $(X, \tau)$ be an $L$-ts. A fuzzy point $x_{\alpha}$ is quasi coincident with $D \in L^{X}$ (and write $x_{\alpha} \prec D$ ) if $x_{\alpha} \not \leq D^{\prime}$. Also $D$ quasi coincides with $D$ at $x(D q E$ at $x)$ if $D(x) \not \leq E^{\prime}(x)$. We say $D$ quasi coincident with $E$ and write $D q E$ if $D q E$ at $x$ for some $x \in X$. Further $D \neg q E$ means $D$ not quasi coincides with $E$. We say $U \in \tau$ is quasi coincident nbd of $x_{\alpha}$ $\left(Q\right.$-nbd) if $x_{\alpha} q U$. The family of all $Q$-nbds of $x_{\alpha}$ is denoted by $Q_{\tau}\left(x_{\alpha}\right)$ or $Q\left(x_{\alpha}\right)$.

Definition 2.6. [19] Let $(X, \tau)$ be an $L$-ts, $D \in L^{X}$ and $\alpha \in M(L)$. $\mathbf{A} \subseteq \tau$ is called an $\alpha$-Q-cover of $D$, if for each $x_{\alpha} \leq D$, there exists $A \in \mathbf{A}$ such that $x_{\alpha} \not \leq A^{\prime} . \mathbf{A}$ is called an $\bar{\alpha}$ - $Q$-cover of $D$, if there exists $\gamma \in \beta^{*}(\alpha)$ such that $\mathbf{A}$ is $\gamma$-Q-cover of $D$.

Definition 2.7. [1] Let $\mathbf{U}=\left\{U_{\lambda}: \lambda \in \Lambda\right\}$, not all zero, be a family of $L$-subsets of an $L$-ts $X$. The order of a fuzzy point $x_{\alpha}$ in $\mathbf{U}$ is the number of elements of $\mathbf{U}$ which are quasi coincident with $x_{\alpha}$. We denote it by Ord $\left(x_{\alpha}, \mathbf{U}\right)$. The order of a collection $\mathbf{U}$ is defined as the largest integer $n$ such that for every $x_{\alpha}$ with $\alpha \in M(L), x_{\alpha}$ quasi coincides with $(n+1)$ members of $\mathbf{U}$. i.e., $\operatorname{Ord}\left(x_{\alpha}, \mathbf{U}\right) \geq n+1$ for all $\alpha \in M(L)$. 
Definition 2.8. A collection $\mathbf{A}$ refines a collection $\mathbf{B}(\mathbf{A}<\mathbf{B})$ if for every $A \in \mathbf{A}$, there exists $B \in \mathbf{B}$ such that $A \leq B$.

Definition 2.9. [19] Let $(X, \tau)$ be an $L$-ts, $\mathbf{A}=\left\{A_{t}: t \in T\right\} \subset L^{X}$, $x_{\lambda} \in M\left(L^{X}\right)$. A is called locally finite at $x_{\lambda}$, if there exist $U \in Q\left(x_{\lambda}\right)$ and a finite subset $T_{0}$ of $T$ such that $t \in T \backslash T_{0} \Rightarrow A_{t} \neg q U$. A is called locally finite for short, if $\mathbf{A}$ is locally finite at every molecule $x_{\lambda} \in M\left(L^{X}\right)$.

Definition 2.10. [19] Let $(X, \tau)$ be an $L$-ts, $A \in L^{X}, \alpha \in M(L)$. $A$ is called $\alpha$-paracompact, if for every open $\alpha$ - $Q$-cover $\Phi$ of $A$ there exist an open refinement $\Psi$ of $\Phi$ such that $\Psi$ is locally finite in $A$ and $\Psi$ is an $\alpha$ $Q$-cover of $A$. $A$ is called paracompact, if $A$ is $\alpha$-paracompact for every $\alpha \in M(L) .(X, \tau)$ is paracompact if $\top$ is paracompact.

Definition 2.11. [1] Let $(X, \tau)$ be an $L$-ts, $D \in L^{X}$. Then $\alpha$-dim $D$ is the least integer $n$ such that every finite open $\alpha$ - $Q$-cover of $D$ has an open $\alpha$ - $Q$-cover refinement of order not exceeding $n$. And $\operatorname{dim} D=n$ if $\alpha$-dim $D=n$ for every $\alpha \in M(L) . \operatorname{dim}(X, \tau)=n$ if $\operatorname{dim} \top=n$.

Definition 2.12. [19] Let $(X, \tau)$ be an $L$-ts, $D \in L^{X}$. $D$ is called $N$ compact if for every $\alpha \in M(L)$, every open $\alpha-Q$ cover of $D$ has a finite sub family which is an $\bar{\alpha}-Q$ - cover of $D$. $(X, \tau)$ is called $N$-compact if $\top$ is $N$-compact.

Definition 2.13. Let $(X, \tau),(Y, \mu)$ be $L$-ts's, $f^{\rightarrow}: L^{X} \rightarrow L^{Y}$ an $L$-fuzzy mapping. Then $f^{\rightarrow}$ is perfect if it is continuous, closed and $f^{\leftarrow}(y)$ is $N$ compact for every $y \in Y$.

\section{Finitistic Spaces}

Definition 3.1. Let $(X, \tau)$ be an $L$-ts, $D \in L^{X}$. $D$ is said to be $\alpha$-finitistic if every $\alpha$ - $Q$-cover of $D$ has an $\alpha$ - $Q$-cover refinement by open $L$-sets with finite order. $D$ is finitistic if $D$ is $\alpha$-finitistic for every $\alpha \in M(L)$. And $(X, \tau)$ is finitistic if $\top$ is finitistic.

Theorem 3.2. Every closed subspace of a finitistic space is finitistic.

Proof. Let $(X, \tau)$ be a finitistic space. And $Y$ be a closed subspace of $X$. Then clearly $Y^{\prime}$ is an open $L$-set in $X$. Let $\mathbf{A}=\left\{A_{\lambda}: \lambda \in \Lambda\right\}$ be an open $\alpha$ - $Q$-cover of $Y$. Then for every $A_{\lambda} \in \mathbf{A}$ there exist open $L$-subsets $B_{\lambda}$ 
of $X$ such that $A_{\lambda}=B_{\lambda} \wedge Y$. Now consider $\mathbf{B}=\left\{B_{\lambda}: A_{\lambda}=B_{\lambda} \wedge Y \forall A_{\lambda} \in\right.$ $\mathbf{A}\} \cup Y^{\prime}$. Then clearly $\mathbf{B}$ is an open $\alpha$ - $Q$-cover of $X$ by open $L$-sets. Since $(X, \tau)$ is finitistic, for every $\alpha \in M(L)$, B has a finite order $\alpha$-Q-cover refinement say $\mathbf{C}$ by open $L$-sets. Then $\mathbf{D}=\{C \wedge Y: C \in \mathbf{C}\}$ is a finite order $\alpha$ - $Q$-cover refinement of $\mathbf{A}$ by open $L$-sets and hence $Y$ is finitistic.

Theorem 3.3. In a weakly induced $L$-ts the following are equivalent:

(i) $(X, \tau)$ is finitistic

(ii) There exist $\alpha \in M(L)$ such that $(X, \tau)$ is $\alpha$-finitistic

(iii) $(X,[\tau])$ is finitistic.

Proof. $\quad$ (i) $\Rightarrow$ (ii) Obvious.

(ii) $\Rightarrow$ (iii): Suppose that there exist $\alpha \in M(L)$ such that $(X, \tau)$ is $\alpha$ finitistic. Let $\mathbf{U}$ be an open cover of $X$. Then $\left\{\chi_{U}: U \in \mathbf{U}\right\}$ is an open $\alpha$ - $Q$-cover of $\top$. Since $(X, \tau)$ is $\alpha$-finitistic, it follows that $\left\{\chi_{U}: U \in \mathbf{U}\right\}$ has an open refinement $\mathbf{V}$ of finite order say $n$.

Now consider $\mathbf{W}=\left\{V_{\left(\alpha^{\prime}\right)}: V \in \mathbf{V}\right\}$ where $V_{\left(\alpha^{\prime}\right)}=\left\{x \in X: V(x) \not \leq \alpha^{\prime}\right\}$. Now by the weakly induced property, $\mathbf{W}$ is an open cover of $X$. Now we will show that $\mathbf{W}$ also has an order not exceeding $n$.

For, if possible, let order of $\mathbf{W}$ be greater than $n$. Therefore there exist $x \in X$ which belongs to at least $n+2$ members of $\mathbf{W}$.

i.e., $x \in\left\{x \in X: V(x) \not \alpha^{\prime}\right\}$ for at least $n+2$ members of $\mathbf{V}$

i.e., $V(x) \not \leq \alpha^{\prime}$ for at least $n+2$ members of $\mathbf{V}$ or $x_{\alpha} \prec V$ for at least $n+2$ members of $\mathbf{V}$. This is contradiction to that order of $\mathbf{V}$ is not exceeding $n$. Hence $(X,[\tau])$ is finitistic.

(iii) $\Rightarrow$ (i): Suppose $(X,[\tau])$ is finitistic. We have to show that $(X, \tau)$ is finitistic.

Let $\mathbf{U} \subset \tau$ be an open $\alpha$ - $Q$-cover of $\top$ where $\alpha \in M(L)$. Since $(X, \tau)$ is weakly induced it follows that $\left\{U_{\left(\alpha^{\prime}\right)}: U \in \mathbf{U}\right\}$ is an open cover of $X$ and it has an open refinement $\mathbf{V}$ of some finite order say $n$. For every $V \in \mathbf{V}$ let $U_{V}$ be such that $V<U_{V\left(\alpha^{\prime}\right)}$. Consider $\mathbf{W}=\left\{\chi_{V} \wedge U_{V}: V \in \mathbf{V}, V<\right.$ $\left.U_{V\left(\alpha^{\prime}\right)}\right\}$. This is an open refinement of $\mathbf{U}$ with order $n$. Hence $(X, \tau)$ is finitistic.

Theorem 3.4. Let $(X, \tau)$ be a finitely supported $L$-ts. Then $(X, \tau)$ is finitistic.

Proof. Let $\mathbf{A}=\left\{A_{\lambda}: \lambda \in \Lambda\right\}$ be an $\alpha$ - $Q$-cover of $(X, \tau)$. Then clearly for each $x_{\alpha} \in M\left(L^{X}\right)$, there exists $A_{x_{\alpha}} \in \mathbf{A}$ such that $x_{\alpha} \not \subset A_{x_{\alpha^{\prime}}}$. Take 
$A_{x}=\left\{\vee A_{x_{\alpha}}: x_{\alpha} \in M\left(L^{X}\right)\right\}$. Since $X$ is finitely supported it follows that the collection $\mathbf{B}=\left\{A_{x}: x \in \operatorname{Supp}(X)\right\} \cup \perp$ is the required finitely ordered open $\alpha$ - $Q$-cover refinement of $\mathbf{A}$ and hence $(X, \tau)$ is finitistic.

Theorem 3.5. Every $N$-Compact $L$-ts $(X, \tau)$ is finitistic.

Proof. Let $(X, \tau)$ be an $N$-Compact space and $\alpha \in M(L)$. Let $\mathbf{A}$ be an $\alpha$ - $Q$-cover of $(X, \tau)$. Since $(X, \tau)$ is $N$-Compact, $\mathbf{A}$ has a finite $\bar{\alpha}-Q$ - cover say $\mathbf{B}_{1}$. Now let $\mathbf{B}=\mathbf{B}_{1} \cup\{\perp\}$. Then it is clear that $\mathbf{B}$ is a finite order open refinement of $\mathbf{A}$. Hence $(X, \tau)$ is finitistic.

Remark 3.6. The converse of theorem 3.5 is not true.

For, let $X$ be any infinite set. Let $\tau=\left\{\chi_{U}: U \subset X\right\}$. Then clearly $(X, \tau)$ is finitistic; because $\mathbf{A}=\left\{\chi_{\{x\}}: x \in X\right\}$ is an open $\alpha$-Q-cover refinement of every open $\alpha$-Q-cover. Now $(X, \tau)$ is not $N$-Compact because $\mathbf{B}=\left\{\chi_{\{x\}}: x \in X\right\}$ is an $\alpha$ - $Q$-cover of $(X, \tau)$ which has no finite subfamily which is an $\bar{\alpha}$ - $Q$-cover.

Now clearly we have the following theorem.

Theorem 3.7. Let $(X, \tau)$ be an $L$-ts such that $\tau$ is finite. Then $(X, \tau)$ is finitistic.

Theorem 3.8. Every fuzzy paracompact space $(X, \tau)$ of finite covering dimension is finitistic.

Proof. Let $(X, \tau)$ be a fuzzy paracompact space with finite covering dimension. Let $\mathbf{A}=\left\{A_{\lambda}: \lambda \in \Lambda\right\}$ be an open $\alpha$ - $Q$-cover of $(X, \tau)$. Since $(X, \tau)$ is fuzzy paracompact, it is $\alpha$ - paracompact for every $\alpha \in M(L)$. Then for every $\alpha$ - $Q$-cover $\mathbf{A}$ of $(X, \tau)$, there exist an open refinement $\mathbf{B}$ of $\mathbf{A}$ such that $\mathbf{B}$ is locally finite in $X$ and $\mathbf{B}$ is $\alpha$ - $Q$-cover of $(X, \tau)$. Now let $\operatorname{dim}(X, \tau)=n$. Then it is clear that $\mathbf{B}$ has an open $\alpha$ - $Q$-cover refinement, say $\mathbf{C}$, of order not exceeding $n$. Hence it follows that $\mathbf{A}$ has an open $\alpha$ - $Q$-cover refinement $\mathbf{C}$ with finite order. Therefore $(X, \tau)$ is finitistic.

\section{Invariant Theorems}

The invariance of finitistic spaces under different types of fuzzy maps will be explored in this section.

Remark 4.1. Continuous image of a finitistic space need not be finitistic. 
Let $\left(X, T_{1}\right)$ be the discrete topological space. Then clearly $\left(X, T_{1}\right)$ is finitistic. Let $\left(X, T_{2}\right)$ be any topological space which is not finitistic. Then it follows that the induced space $\left(X, \omega\left(T_{1}\right)\right)$ is finitistic. But $\left(X, \omega\left(T_{2}\right)\right)$ is not finitistic. Let $f:\left(X, \omega\left(T_{1}\right)\right) \rightarrow\left(X, \omega\left(T_{2}\right)\right)$ be defined as $f(x)=x$, for all $x \in X$. Then clearly $f$ is continuous and onto. i.e., $\left(X, \omega\left(T_{2}\right)\right)$ is continuous image of $\left(X, \omega\left(T_{1}\right)\right)$ where $\left(X, \omega\left(T_{1}\right)\right)$ is finitistic but $\left(X, \omega\left(T_{2}\right)\right)$ is not finitistic.

Result 4.2. If $(X, \tau),(Y, \mu)$ are two weakly induced $L$-topological spaces, then

(i) If the map $f^{\rightarrow}: L^{X} \rightarrow L^{Y}$ is $L$-fuzzy continuous, then $f:(X,[\tau]) \rightarrow$ $(Y,[\mu])$ is continuous

(ii) If the map $f^{\rightarrow}: L^{X} \leftarrow L^{Y}$ is $L$-fuzzy closed, then $f:(X,[\tau]) \rightarrow(Y,[\mu])$ is closed

(iii) If the map $f^{\rightarrow}: L^{X} \rightarrow L^{Y}$ is $L$-fuzzy open, then $f:(X,[\tau]) \rightarrow(Y,[\mu])$ is open.

Theorem 4.3. Let $(X, \tau),(Y, \mu)$ are two weakly induced $L$-topological spaces. Then if $f^{\rightarrow}: L^{X} \rightarrow L^{Y}$ is perfect, then so is $f:(X,[\tau]) \rightarrow(Y,[\mu])$.

Proof. Let $y_{\alpha} \in L^{Y}$. Since $f^{\rightarrow}: L^{X} \rightarrow L^{Y}$ is perfect, $f^{\leftarrow}\left(y_{\alpha}\right)$ is $N$ compact. Now to prove $f:(X,[\tau]) \rightarrow(Y,[\mu])$ is perfect, it is enough to prove that $f^{\leftarrow}\left(y_{\alpha}\right)$ is compact for every $y \in Y$. Now let $\mathbf{U} \in[\tau]$ be an open cover of $f^{\leftarrow}(y)$. Consider $\mathbf{U}^{*}=\left\{\chi_{U}: U \in \mathbf{U}\right\}$. This is clearly an open $\alpha$ $Q$-cover of $f^{\leftarrow}\left(y_{\alpha}\right)$. For, let $x_{\alpha} \leq f^{\leftarrow}\left(y_{\alpha}\right)$ i.e., $f^{\leftarrow}\left(y_{\alpha}\right)(x)=y_{\alpha}(f(x)) \geq \alpha$. Now let $U \in \mathbf{U}$ be such that $x \in U$. This is possible since $U$ is a cover of $f^{\leftarrow}(y)$. Then $\chi_{U}(x) \geq y_{\alpha}(x) \geq \alpha$. i.e., $\chi_{U}(x) \geq \alpha$ or $x_{\alpha} \leq \chi_{U}$. Hence clearly $x_{\alpha} q \chi_{U}$. Hence $\left\{\chi_{U}: U \in \mathbf{U}\right\}$ is an open $\alpha$-Q-cover of $f^{\leftarrow}\left(y_{\alpha}\right)$.

Again $f^{\leftarrow}\left(y_{\alpha}\right)$ being $N$-compact, there exists a finite sub collection $\mathbf{U}_{s}^{*}$ of $\mathbf{U}^{*}$ which is also an $\bar{\alpha}$-Q-cover of $f^{\leftarrow}\left(y_{\alpha}\right)$. Let $\mathbf{U}_{s}^{*}=\left\{\chi_{U 1}, \chi_{U 2}, \ldots, \chi_{U k}\right\}$. Then clearly $\left\{U_{1}, U_{2}, \ldots, U_{k}\right\}$ will be a finite sub cover of $f^{\leftarrow}(y)$. This completes the proof.

Now by Theorem 3.3 we readily have

Theorem 4.4. $(X, \tau),(Y, \mu)$ are two weakly induced $L$-ts's and $f \rightarrow$ : $L^{X} \rightarrow L^{Y}$ be a perfect map. Then $(X, \tau)$ is finitistic if and only if $(Y, \mu)$ is finitistic.

\section{Acknowledgements}

Authors are very much indebted to Prof. T. Thrivikraman, Formerly Department of Mathematics, Cochin University of Science and Technology, 
India for his constant encouragement in the preparation of this paper.

\section{References}

[1] T. Baiju and Sunil Jacob John, Covering dimension and Normality in $L$-topological spaces, (Communicated).

[2] G. E. Bredon, Introduction to Compact Transformation Groups, Academic Press, (1972).

[3] C. Chang, Fuzzy Topological Spaces, Journal of Math. Anal. Appl., Vol. 24, pp. 182-190, (1968).

[4] S. Deo, Topology of finitistic spaces and related topics, Bull. Allahabad Math. Soc., Vol. 2, pp. 31-61, (1987).

[5] S. Deo and A. R. Pears, A completely finitistic space is finite dimensional, Bull. London Math. Soc., Vol. 17, pp. 49-51, (1985).

[6] S. Deo and M. Singh, On certain construction in finitistic spaces, Int. J. Math. And Math. Soc. Vol. 6, pp. 477-482, (1983).

[7] S. Deo and H. S. Tripathy, Compact lie group action on finitistic spaces, Topology, Vol. 21, pp. 393-399, (1982).

[8] J. Goguen, L-fuzzy Sets, J. Math. Anal. Appl., Vol. 18, pp. 145-174, (1967).

[9] Y. Hattori, A note on finitistic spaces, Q \& A in General Topology, Vol. 3, pp. 43-55, (1985).

[10] Y. Hattori, finitistic spaces and Dimension, Houston Journal of Mathematics, Vol. 25, No. 4, (1999).

[11] U. Hohle and S. E. Rodabaugh, Mathematics of Fuzzy Sets : Logic, Topology and Measure Theory, The Hand Book of Fuzzy Set Series 3, Kluwer Academic Pub., (1999).

[12] D. S. Jamwal and Shakeel Ahmed, Covering Dimension and Finitistic Spaces in L-topology, Conf. Proc. Fuzzy Set Theory, held in B.H.U., Allied Pub., pp. 117-122, (2002). 
[13] T. Kubiak, The topological modification of the L-fuzzy unit interval, : S.E. Rodabaugh, E.P. Klement, U. Hohle (Eds.), Applications of Category Theory to Fuzzy Subsets, Kluwer Academic Publishers, Dordrecht, pp. 275-305,(1992).

[14] R. Lowen, Fuzzy topological spaces and fuzzy compactness, J. Math. Anal. Appl., Vol. 56, pp. 621-633, (1976).

[15] Shakeel Ahmed, On $\alpha$-Finitistic Spaces, Tamsui Oxford Journal of Mathematical Sciences, Vol. 22, No. 1, pp. 73-82, (2006).

[16] R. G. Swan, A new method of fixed point theory, Comm. Math. Helv. Vol. 34, pp. 1-16, (1960).

[17] G. J. Wang, On the structure of fuzzy lattices, Acta math. Sinica, Vol. 29, pp. 539-543, (1986).

[18] G. J. Wang, Theory of $L$-fuzzy topological spaces, Shaanxi Normal University Pub., Xian, (1988).

[19] Ying-Ming Liu and Mao-Kang Luo, Fuzzy Topology, Advances in Fuzzy SystemsApplications and Theory Vol.9, World Scientific, (1997).

\author{
T. Baiju \\ Department of Mathematics \\ National Institute of Technology Calicut \\ Calicut-673, 601, \\ Kerala, \\ India \\ e-mail : bethelbai@yahoo.co.in, \\ and \\ Sunil Jacob John \\ Department of Mathematics \\ National Institute of Technology Calicut \\ Calicut-673, 601, \\ Kerala, \\ India \\ e-mail : sunil@nitc.ac.in
}

